\title{
¿A una empresa le conviene endeudarse con el banco?
}

\section{RESUMEN}

Las empresas cuentan con múltiples alternativas de financiamiento que requieren ser analizadas para definir la combinación adecuada entre deuda y el aporte de capital del accionista (o accionistas). El modelo financiero basado en el VPN como criterio de decisión ayuda a seleccionar la mejor alternativa de financiamiento con deuda. La deuda bancaria mejora el valor de la empresa, pero se requiere un equilibrio entre endeudamiento y capital propio. La deuda bancaria es conveniente en la medida que la tasa de interés sea inferior al costo de oportunidad del accionista. El beneficio de la deuda para el accionista se da cuando la empresa contabiliza los gastos financieros en la determinación de la participación de los trabajadores en las utilidades de la empresa y en el pago del impuesto a la renta a la SUNAT.

Palabras Clave: Valor de una empresa, sistema bancario, tasa de interés, costo de oportunidad, valor presente neto.

IS IT CONVENIENT FOR AN ENTERPRISE TO FALL INTO DEBT WITH A BANK?

ABSTRACT

Enterprises count on multiple financing alternatives which deserve to be analized in order to come out the most suitable combination between a debt and the shareholder (or shareholders) capital contribution. The financial model based on VPN as a decision criterium helps to select the best financial alternative with a debt. Banking debt improves the enterprise's value, but a balance between indebtedness and own capital is required. Banking debt can be convenient as interest rate goes below the shareholder's opportunity cost . Debt benefit for the shareholder shows up when the enterprise enters financial expenditures in determining workers shares within the enterprise's utilities and the SUNAT's income tax payment in the books.

Key Words: Enterprise's Value, Banking System, Interest Rate, Opportunity Cost, Net Present Value.

\section{INTRODUCCIÓN}

Las empresas para cubrir sus necesidades de fondos (recursos financieros) requieren financiarse con capital de sus accionistas (socios) y con recursos de terceros (bancos, proveedores y otras fuentes). Estas necesidades financieras obedecen a planes de crecimiento, modernización, diversificación de actividades, o de fortalecimiento de la organización, y comprenden la ejecución de estudios, y adquisiciones de activos fijos y/o de capital de trabajo.

Entre las diversas fuentes de fondos de terceros que las empresas utilizan, la deuda bancaria es la más común. Para una empresa, utilizar el crédito (préstamo) del banco para financiar sus inversiones, permite un menor aporte de capital de sus accionistas y los gastos que demande dicha deuda (intereses y otros cargos relacionados), la empresa los contabiliza como gastos financieros.

Es de esperar que, bajo determinadas condiciones, la deuda bancaria mejore la rentabilidad de la empresa, básicamente por el doble efecto de la deuda, en primer término le permite al accionista desarrollar el negocio empresarial con menor aporte de capital y en segundo término por el hecho que los intereses de la deuda permiten a la empresa menores pagos de participación de utilidades a trabajadores y de impuesto a la renta, dando lugar a mayor disponibilidad de caja para sus accionistas.

En este contexto, el objetivo del presente artículo consiste en definir las condiciones básicas bajo las cuales la deuda bancaria es conveniente para una empresa, teniendo en cuenta que su finalidad es maximizar el valor de las acciones representativas del capital social de la empresa.

Los resultados que se exponen facilitan el diseño e implantación de una estrategia financiera que contribuya a un crecimiento equilibrado de la empresa, lo que no solo maximizará el valor de la empresa para sus accionistas, sino también para sus trabajadores y la sociedad.

Los alcances del artículo son aplicables para una empresa formal que tiene posibilidades de acceso al crédito bancario y que está afecta al pago de participación de utilidades a sus trabajadores y se encuentra bajo el régimen general del impuesto a la renta de la Superintendencia Nacional de Administración Tributaria (SUNAT).

\section{VALOR DE UNA EMPRESA}

El éxito de una organización empresarial depende de la habilidad de sus integrantes para relacionarse con su entorno o medio ambiente y

\footnotetext{
1) Magíster en Administración de Empresas. Profesor del Departamento de Producción y Gestión Industrial, UNMSM E-mail: nestor_sj@hotmail.com
} 
para optimizar la cadena de valor de la empresa, la cual se puede representar como una matriz insumoproducto, que consta de tres instancias básicas:

\section{- Mercado de proveedores}

Donde la empresa se suministra de los diversos insumos que requiere para producir y comercializar sus productos, sean bienes 0 servicios.

\section{- Proceso operativo}

Constituido por la tecnología y la gestión del negocio, que comprende diversos procedimientos y normas, cuya finalidad es generar valor agregado para la empresa, obteniendo los productos demandados por sus clientes.

\section{- Mercado de consumidores}

Representado por distribuidores, clientes y competidores, donde los clientes requieren satisfacer sus necesidades en términos de calidad, oportunidad y precio.

Desde el punto de vista financiero, el éxito de una empresa se refleja por el flujo de dinero, en donde los ingresos por las cobranzas de las ventas, permiten atender todos los pagos por diversas obligaciones, sean éstas operativas, financieras o tributarias. Adicionalmente, el saldo neto (ingresos menos egresos) de dicho flujo permite compensar las expectativas de ganancia de los accionistas y generar excedentes para seguir desarrollando con éxito la actividad empresarial.

El dinero (medido a través del flujo de caja) no es suficiente para decidir sobre la inversión a ejecutar, sino que se requiere constancia, dedicación de los integrantes de la organización y buenas relaciones de la empresa con su entorno. La sostenibilidad de la empresa como negocio en marcha es fundamental, durante un horizonte que facilite la toma de decisiones cruciales, como por ejemplo, continuar o no en el negocio, crecer en el giro del negocio o diversificar en otras actividades empresariales.

Por lo tanto, bajo determinadas premisas es posible desarrollar y ejecutar un modelo financiero, que contribuya a tomar decisiones según las alternativas que se definan, orientado a maximizar el valor de la empresa. Este modelo requiere de dos instrumentos básicos de análisis:

\section{- Flujo de caja proyectado del negocio empresarial}

Donde se evidencie el saldo neto de efectivo que le correspondería al accionista según las premisas asumidas para la proyección de los ingresos y los egresos de caja de la empresa.

\section{- Costo de oportunidad del accionista}

Es decir la tasa de interés expresada en la misma moneda y periodo que del flujo de caja, que represente el retorno o rendimiento mínimo que el accionista espera por el capital aportado a la empresa.

El análisis del flujo de caja con el costo de oportunidad dará lugar a un monto equivalente a una fecha determinada, el cual recibe la denominación del Valor Presente Neto (VPN).

En otros términos, el VPN es una ganancia monetaria equivalente del flujo de caja proyectado, el cual es actualizado a la tasa del costo de oportunidad. En tal sentido, el efecto de la deuda bancaria para el accionista, se puede apreciar mediante el VPN. En principio, alguna alternativa de deuda que se considere para el financiamiento de la empresa debe aumentar el VPN. Entre dos alternativas de deuda, la más conveniente será aquella con mayor VPN.

No cabe duda que un buen análisis de las alternativas de decisión sobre la deuda bancaria, además de la adecuada estimación del flujo de caja, depende del costo de oportunidad del accionista. El valor de esta tasa está representado por el rendimiento de la mejor alternativa sacrificada por el accionista al comprometer su capital en la empresa, al cual se le debe adicionar una prima por riesgo, es decir una cobertura porcentual ante una posible pérdida del capital invertido en el negocio empresarial.

\section{COSTO DEL CRÉDITO BANCARIO}

Existen diversos factores que una empresa debe considerar para endeudarse. Uno de los factores que no debe faltar en el análisis es la tasa de interés de la deuda, especialmente en un mercado donde estas tasas son libres, es decir lo fija la oferta y la demanda de dinero, según el estrato o segmento que sea de interés para las entidades financieras.

Para garantizar un proceso adecuado de negociación entre la banca (que otorga el crédito) y las empresas (que requieren endeudarse), la Superintendencia de Banca, Seguros y AFP (SBS) ha establecido mecanismos de transparencia de la información sobre las condiciones de los créditos bancarios. En la actualidad el sistema financiero nacional cuenta con once bancos.

De esta manera, todos los días la SBS publica las tasas de interés promedio que las entidades del sistema bancario (denominado también Banca Múltiple) vienen aplicando por los créditos que otorgan. Se publica un promedio global por moneda, tipo de operación y por entidad financiera. 
A una fecha determinada se tiene la TAMN y la TAMEX, que son las tasas de interés activas promedio del sistema bancario, para créditos en moneda nacional ( $\mathrm{MN}$ ) y para créditos en moneda extranjera (ME). Adicionalmente, se tiene el promedio de los últimos 30 días útiles de la tasa de interés del sistema bancario, detallado por tipo de operaciones realizadas:

\section{- Crédito comercial}

Son operaciones con las empresas medianas y grandes, comúnmente denominados por los bancos como clientes corporativos, así como clientes que superan los límites establecidos para la microempresa.

\section{- Crédito a microempresas}

Corresponde a créditos otorgados a las personas naturales o jurídicas que cuenten con activos (sin considerar inmuebles) no mayores a US\$20 000 y deudas que no exceden el mismo monto o su equivalente en moneda nacional.

\section{- Créditos de consumo}

Incluye los créditos de la banca personal y de la banca de consumo, y a las tarjetas de créditos.

\section{- Crédito hipotecario}

Son los préstamos para la adquisición de inmuebles.

En el Cuadro 1 se presentan las tasas de interés pasivas del sistema bancario al 30 de setiembre de 2006, por tipo de moneda y por tipo de crédito (promedio de los últimos 30 días útiles) y el promedio del sistema a dicha fecha.

Se puede apreciar una variación importante de las tasas de interés según las operaciones (el menor costo corresponde al crédito comercial y el mayor al crédito de consumo). El promedio del sistema bancario es muy referencial y no refleja las diferencias en las tasas, según tipos de operación, montos, plazos, clientes y entidades bancarias.

Por ejemplo, en el crédito comercial, se tiene un detalle de operaciones que se identifican de la siguiente manera: avances en cuentas corrientes; sobregiros; y descuentos y préstamos menores a 30 días, entre 31 y 90 días, 91 y 180 días, 181 y 360 días, y más de 360 días.

El costo de la deuda, en este caso representado por la tasa de interés, va a ser objeto de evaluación, para medir su grado de impacto en los resultados de la empresa, y por ende en el VPN, es decir la ganancia neta esperada por sus accionistas.

La ventaja de la deuda bancaria para una empresa formal, es que los intereses se contabilizan como gastos financieros, por lo que en el estado de ganancias y pérdidas se registrarán menores pagos de participación de trabajadores y de impuesto a la renta, proceso que se denomina "ahorro fiscal", dado que se traduce en una mejora en el saldo neto de caja de la empresa durante el periodo de vigencia del crédito, con lo cual se tiene un aumento en el VPN.

\section{APALANCAMIENTO FINANCIERO}

Otro aspecto de relevancia en el análisis del efecto de la deuda en el VPN de una empresa, es el nivel de deuda asumido con relación al aporte de capital del accionista, comúnmente llamado grado de apalancamiento o de endeudamiento empresarial, y que se mide por el cociente entre el pasivo (deudas) y el patrimonio neto (porción de la Empresa que es de propiedad de los accionistas).

A mayor relación entre deuda y capital propio (D/C), el accionista compromete menos capital para desarrollar su empresa, el cual sería deseable en la medida que existiese seguridad en el compromiso de devolución de la deuda. A mayor relación $D / C$ en principio se espera un mayor valor del VPN. Un análisis completo requiere relacionar $\mathrm{D} / \mathrm{C}$ con el costo de la deuda y el costo de oportunidad del accionista.

Toda vez que el VPN representa el valor equivalente en el momento actual del flujo de caja de la empresa, en los resultados del análisis influye la forma cómo se paga la deuda, por ejemplo, en una sola cuota de capital, en varias cuotas de capital, en cuotas de

Cuadro 1. Tasas de Interés Promedio del Sistema Bancario al 30 de Setiembre de 2006 (En \% anual efectiva)

\begin{tabular}{|l|c|c|c|c|c|}
\hline \multirow{2}{*}{ Tipo de Moneda } & \multicolumn{4}{|c|}{ Tipo de Crédito } & Promedio del \\
\cline { 2 - 6 } & Comercial & Microempresas & Consumo & Hipotecario & $\mathbf{2}$ \\
\hline Moneda Nacional & $\mathbf{9 , 1 2} \%$ & $39,20 \%$ & $40,84 \%$ & $10,01 \%$ & $\mathbf{2 3 , 7 4 \%}$ \\
\hline Moneda Extranjera & $\mathbf{9 , 7 0} \%$ & $26,05 \%$ & $17,13 \%$ & $9,62 \%$ & $\mathbf{1 0 , 6 7 \%}$ \\
\hline
\end{tabular}

Fuente: SBS 
capital constante, en cuotas totales constantes, etc.

El límite a las condiciones del financiamiento, incluso de la tasa de interés y de la forma de pago de la deuda, en un mercado reducido como el sistema bancario nacional, lo establecen los bancos. Sin embargo, dependiendo del tipo de cliente, monto de la operación y el giro del negocio, los bancos tienden a ser flexibles en el proceso de negociación. El criterio básico es que el financiamiento tiene que ser satisfactorio para ambas partes: banco y empresa.

\section{RESULTADOS ESPERADOS}

Para analizar el efecto de la deuda bancaria en el valor de una empresa que asume dicha obligación, se ha aplicado el siguiente procedimiento:

\section{Información base}

Se tiene un caso hipotético de una empresa que requiere invertir S/.120 000 en activos fijos, para obtener una ganancia operativa en efectivo (antes de depreciación, gastos financieros y deducción de participación de trabajadores y de impuesto a la renta) de S/.45 000; S/.65 000; y S/.85 000 en el primer, segundo y tercer año de operación comercial del negocio, respectivamente.

Dicha inversión se deprecia a la tasa de $20 \%$ anual y el valor residual de la inversión (que se obtiene al año siguiente al periodo de operación comercial de la empresa) equivale al valor en libros contables de la empresa.

El $60 \%$ de la inversión se financia con deuda bancaria, a la tasa de interés de $10 \%$ anual, a ser pagada en tres cuotas anuales de capital constantes, en un plazo total de tres años. La empresa está afecta al pago de impuesto a la renta de $30 \%$ sobre la utilidad anual luego de deducir el $10 \%$ por concepto de participación de utilidades a sus trabajadores. Asimismo, el accionista de esta empresa requiere por su aporte un retorno mínimo de $15 \%$ anual.

Cuadro 2. Estado de Ganancias y Pérdidas y Flujo de Caja

\begin{tabular}{|c|c|c|c|c|}
\hline Concepto & 1 & 2 & 3 & 4 \\
\hline Ganancia Operativa en Efectivo & 45000 & 65000 & 85000 & \\
\hline (-) Depreciación & 24000 & 24000 & 24000 & \\
\hline (-) Gastos Financieros & 0 & 0 & 0 & \\
\hline Utilidad antes Part. Trab. e I. Renta & 21000 & 41000 & 61000 & \\
\hline (-) Participación de Trabajadores & 2100 & 4100 & 6100 & \\
\hline (-) Impuesto a la Renta & 5670 & 11070 & 16470 & \\
\hline Utilidad Neta & 13230 & 25830 & 38430 & \\
\hline
\end{tabular}

\begin{tabular}{|c|c|c|c|c|c|}
\hline Concepto & 0 & 1 & 2 & 3 & 4 \\
\hline $\begin{array}{l}\text { Ingresos: } \\
\text { Ganancia Operativa en Efectivo } \\
\text { Desembolso de Deuda } \\
\text { Valor Residual }\end{array}$ & 0 & 45000 & 65000 & 85000 & 48000 \\
\hline Total Ingresos & 0 & 45000 & 65000 & 85000 & 48000 \\
\hline $\begin{array}{l}\text { Egresos: } \\
\text { Inversión } \\
\text { Principal de Deuda } \\
\text { Gastos Financieros } \\
\text { Participación de Trabajadores } \\
\text { Impuesto a la Renta }\end{array}$ & 120000 & $\begin{array}{r}0 \\
0 \\
2100 \\
5670\end{array}$ & $\begin{array}{r}0 \\
0 \\
4100 \\
11070\end{array}$ & $\begin{array}{rr} & 0 \\
0 \\
6100 \\
16470\end{array}$ & \\
\hline Total Egresos & 120000 & 7770 & 15170 & 22570 & $\mathbf{0}$ \\
\hline Saldo Neto del Periodo & -120000 & 37230 & 49830 & 62430 & 48000 \\
\hline
\end{tabular}


Cuadro 3. Estado de Ganancias y Pérdidas y Flujo de Caja

\begin{tabular}{|l|r|rrr|r|}
\hline \multicolumn{1}{|c|}{ Concepto } & $\mathbf{0}$ & $\mathbf{1}$ & $\mathbf{2}$ & $\mathbf{3}$ & $\mathbf{4}$ \\
\hline Ganancia Operativa en Efectivo & & 45000 & 65000 & 85000 & \\
(-) Depreciación & & 24000 & 24000 & 24000 & \\
(-) Gastos Financieros & & 7200 & 4800 & 2400 & \\
\hline Utilidad antes Part. Trab. e I. Renta & & 13800 & 36200 & 58600 & \\
\hline (-) Participación de Trabajadores & & 1380 & 3620 & 5860 & \\
(-) Impuesto a la Renta & & 3726 & 9774 & 15822 & \\
\hline Utilidad Neta & & $\mathbf{8 6 9 4}$ & $\mathbf{2 2 ~ 8 0 6}$ & $\mathbf{3 6 ~ 9 1 8}$ & \\
\hline
\end{tabular}

\begin{tabular}{|l|r|rrr|r|}
\hline \multicolumn{1}{|c|}{ Concepto } & $\mathbf{0}$ & $\mathbf{1}$ & $\mathbf{2}$ & $\mathbf{3}$ & $\mathbf{4}$ \\
\hline Ingresos: & & & & & \\
Ganancia Operativa en Efectivo & & 45000 & 65000 & 85000 & \\
Desembolso de Deuda & 72000 & & & & \\
Valor Residual & & & & & 48000 \\
\hline Total Ingresos & $\mathbf{7 2 0 0 0}$ & $\mathbf{4 5 0 0 0}$ & $\mathbf{6 5 0 0 0}$ & $\mathbf{8 5 0 0 0}$ & $\mathbf{4 8 0 0 0}$ \\
\hline Egresos: & & & & & \\
Inversión & 120000 & & & & \\
Principal de Deuda & & 24000 & 24000 & 24000 & \\
Gastos Financieros & & 7200 & 4800 & 2400 & \\
Participación de Trabajadores & & 1380 & 3620 & 5860 & \\
Impuesto a la Renta & & 3726 & 9774 & 15822 & \\
\hline Total Egresos & $\mathbf{1 2 0 0 0 0}$ & $\mathbf{3 6} \mathbf{3 0 6}$ & $\mathbf{4 2 1 9 4}$ & $\mathbf{4 8 0 8 2}$ & $\mathbf{0}$ \\
\hline Saldo Neto del Periodo & $\mathbf{- 4 8 0 0 0}$ & $\mathbf{8 6 9 4}$ & $\mathbf{2 2 8 0 6}$ & $\mathbf{3 6 ~ 9 1 8}$ & $\mathbf{4 8 0 0 0}$ \\
\hline
\end{tabular}

\begin{tabular}{|l|}
\hline VPN 28523 \\
\hline
\end{tabular}

Cuadro 4. Relación D/C y Tasa de Interés (Costo de Oportunidad: 15\% anual)

\begin{tabular}{|c|c|c|c|c|c|c|c|}
\hline & & \multicolumn{6}{|c|}{ \%Deuda de la Inversión } \\
\hline & & $0 \%$ & $25 \%$ & $50 \%$ & $60 \%$ & $80 \%$ & $90 \%$ \\
\hline \multirow{6}{*}{$\begin{array}{l}\text { Tasa de Interés } \\
\text { ( } \% \text { anual) }\end{array}$} & $0 \%$ & 18545 & 25713 & 32881 & 35748 & 41482 & 44349 \\
\hline & $5 \%$ & 18545 & 24208 & 29870 & 32135 & 36666 & 38931 \\
\hline & $10 \%$ & 18545 & 22703 & 26860 & 28523 & 31849 & 33512 \\
\hline & $15 \%$ & 18545 & 21198 & 23850 & 24910 & 27032 & 28093 \\
\hline & $20 \%$ & 18545 & 19692 & 20839 & 21298 & 22215 & 22649 \\
\hline & $25 \%$ & 18545 & 18187 & 17829 & 17685 & 17273 & 17003 \\
\hline
\end{tabular}

Cuadro 5. Relación D/C y Costo de Oportunidad (Tasa de Interés: 10\% anual)

\begin{tabular}{|r|r|r|r|r|r|r|r|}
\cline { 3 - 8 } \multicolumn{2}{c|}{} & \multicolumn{6}{c|}{ \%Deuda de la Inversión } \\
\cline { 3 - 8 } \multicolumn{2}{c|}{} & \multicolumn{1}{c|}{$\mathbf{0 \%}$} & $\mathbf{2 5 \%}$ & $\mathbf{5 0 \%}$ & $\mathbf{6 0 \%}$ & $\mathbf{8 0 \%}$ & $\mathbf{9 0 \%}$ \\
\hline & $\mathbf{0 \%}$ & 77490 & 73710 & 69930 & 68418 & 65394 & 63882 \\
\cline { 2 - 8 } & $\mathbf{1 0 \%}$ & 34717 & 36615 & 38514 & 39273 & 40792 & 41552 \\
\cline { 2 - 8 } Costo de Oportunidad & $\mathbf{1 5 \%}$ & 18545 & 22703 & 26860 & $\mathbf{2 8 5 2 3}$ & 31849 & 33512 \\
\cline { 2 - 8 }$(\%$ anual) & $\mathbf{2 0 \%}$ & 4906 & 11026 & 17147 & 19595 & 24492 & 26940 \\
\cline { 2 - 8 } & $\mathbf{2 5 \%}$ & -6700 & 1139 & 8978 & 12114 & 18385 & 21521 \\
\cline { 2 - 8 } & $\mathbf{3 0 \%}$ & -16654 & -7301 & 2051 & 5792 & 13274 & 17016 \\
\hline
\end{tabular}


Cuadro 6. Costo de Oportunidad y Tasa de Interés (\%Deuda de la Inversión: 60\%)

\begin{tabular}{|c|c|c|c|c|c|c|c|}
\hline & & \multicolumn{6}{|c|}{ Costo de Oportunidad ( $\%$ anual) } \\
\hline & & $0 \%$ & $10 \%$ & $15 \%$ & $20 \%$ & $25 \%$ & $30 \%$ \\
\hline \multirow{6}{*}{$\begin{array}{r}\text { Tasa de Interés } \\
\text { (\% anual) }\end{array}$} & $0 \%$ & 77490 & 47032 & 35748 & 26350 & 18452 & 11759 \\
\hline & $5 \%$ & 72954 & 43153 & 32135 & 22973 & 15283 & 8776 \\
\hline & $10 \%$ & 68418 & 39273 & 28523 & 19595 & 12114 & 5792 \\
\hline & $15 \%$ & 63882 & 35394 & 24910 & 16218 & 8945 & 2809 \\
\hline & $20 \%$ & 59346 & 31514 & 21298 & 12840 & 5776 & -174 \\
\hline & $25 \%$ & 54810 & 27635 & 17685 & 9463 & 2606 & -3158 \\
\hline
\end{tabular}

\section{VPN sin considerar deuda}

Con los datos considerados la ganancia esperada por los accionistas, medida en términos de VPN, es S/.18 545 a valores del año cero y para un costo de oportunidad (tasa de descuento de 15\% anual), según estimados que se aprecian el en Cuadro 2. Como se podrá observar no hay gastos financieros en el Estado de ganancias y pérdidas; y en el flujo de caja, no hay desembolso de deuda de parte del banco y por lo tanto no existe servicio de deuda (principal más intereses).

\section{VPN considerando deuda}

Si la empresa asume la deuda bancaria, en las condiciones planteadas, el VPN mejora a S/.28 523, es decir aumenta en $54 \%$ con respecto a ejecutar la inversión sólo con el aporte de capital del accionista.

Según las proyecciones del Cuadro 3, este beneficio se debe al efecto del apalancamiento, es decir el accionista, para el mismo negocio aporta menos capital; y por el hecho que se tienen menos egresos por participación de trabajadores y de impuesto a la renta (en el estado de ganancias y pérdidas), lo cual implica menores salidas (en el flujo de caja), dando lugar al aumento en el VPN.

Hasta aquí se verifica que el VPN aumenta cuando una empresa asume deuda bancaria. A continuación vamos a ver cuáles son los límites de este beneficio en la mejora del valor de la empresa.

\section{Efectos de la tasa de interés y del apalancamiento financiero}

En el Cuadro 4, el VPN para un costo de oportunidad de $15 \%$ anual, mejora para mayores niveles de endeudamiento, en la medida que la tasa de interés de la deuda sea igual o menor a $10 \%$ anual. $A$ mayores tasas de interés, el mayor porcentaje de endeudamiento implica una reducción en el VPN con respecto al financiamiento sólo con capital propio.

Esta bondad de la deuda será tangible si los ingresos netos en efectivo (diferencia entre ingresos por ventas y pagos por obligaciones operativas) se presentan en los niveles proyectados, caso contrario el riesgo de incumplimiento de la deuda aumenta y la situación mostrada de favorable-se vuelve perjudicial para el accionista, quien recibe compensación según los resultados del éxito de su negocio luego de cumplir todas sus obligaciones.

\section{Efectos del apalancamiento financiero y del costo de oportunidad}

Para una tasa de interés de la deuda de $10 \%$ anual, en el Cuadro 5, se tienen las variaciones en el VPN para diferentes valores del costo de oportunidad y del $\%$ de deuda que financia a la inversión de la empresa. Como era de esperase a mayor tasa del costo de oportunidad, el VPN disminuye, e incluso el beneficio de mayor endeudamiento no compensa este deterioro en el valor de la empresa.

\section{Efectos de la tasa de interés y del costo de oportunidad del accionista}

En el Cuadro 6 se tiene la variación del VPN, para una deuda que cubre el $60 \%$ de la inversión total, ante cambios en la tasa de interés de la deuda y del costo de oportunidad.

EI VPN es menos sensible a los cambios en la tasa de interés, que a las variaciones en el costo de oportunidad y del apalancamiento financiero. Tasas de interés elevadas de la deuda, en cierta medida no impactan de manera crítica en el valor de la empresa, que las mayores exigencias de los accionistas (alta tasa del costo de oportunidad) y el riesgo de incumplimiento de pago por asumir mayores niveles de deuda con relación al aporte de capital propio del accionista.

\section{CONCLUSIONES}

Para una adecuada decisión de endeudamiento se requiere definir el costo de oportunidad del accionista, el cual debe incluir una prima por riesgo ante un posible incumplimiento del pago de la deuda derivada de cambios en el escenario de proyección de la empresa analizada. 
Los administradores de las empresas deben mantener una permanente búsqueda de créditos bancarios con adecuados costos y facilidades de pago, para considerar un nivel de deuda que mejore el valor de la empresa, pero sin arriesgar las expectativas de ganancia del accionista. La tasa de interés de la deuda tiene que ser sustancialmente menor que el costo de oportunidad del accionista.

Toda vez que la decisión de endeudarse se base en premisas del escenario futuro en que se desarrollará la empresa, es conveniente contar con mecanismos de respuesta antes cambios no previstos, para reducir la exposición a la deuda, y así no afectar el valor de las acciones de la empresa para sus accionistas, sus trabajadores y la sociedad.

\section{REFERENCIAS BIBLIOGRÁFICAS}

1. Fernández, P. (2002). Valoración de empresas. Segunda Edición. Edic. Gestión 2000, España.

2. Moyer, R. Ch., Mcguigan, J.R., Kretlow, W.J. (1998). Administración financiera contemporánea. Séptima edición, International Thomson Editores, México.

3. Superintendencia de Banca, Seguros y AFP (2006). Tasas de interés activas en el sistema bancario. En:

http://www.sbs.gob.pe/portalsbs/tipotasa/indice. htm

(Visitado: 02-10-06) 